\title{
Electrokinetic delivery of reactants: pore water chemistry controls transport, mixing and degradation
}

\section{Supporting Information}

\author{
Riccardo Sprocati ${ }^{1}$, Andrea Gallo ${ }^{2}$, Rajandrea Sethi ${ }^{2}$, Massimo Rolle $^{1^{*}}$ \\ ${ }^{1}$ Department of Environmental Engineering, Technical University of Denmark, \\ Bygningstorvet, Building 115, 2800 Kgs. Lyngby, Denmark \\ ${ }^{2}$ Department of Environmental, Land and Infrastructure Engineering, Politecnico di Torino, \\ Corso Duca degli Abruzzi 24, 10129 Torino, Italy
}

*Corresponding author phone: +45 45251566; email: masro@env.dtu.dk

Number of Pages: 12

Number of Figures: 6

Number of Tables: 4 


\section{S1. Details of the experimental setup}

Figure S1 shows the two experimental setups used for the advection-dispersion (Figure S1a) and EK (Figure S1b) scenarios.

For each experiment we used the same type of glass beads as porous medium. The glass beads were washed with a solution of $5 \%$ hydrogen peroxide and $5 \% \mathrm{NaCl}$ for at least 8 hours and successively rinsed with at least 10 pore volumes of deionized water and 5 pore volumes of ultrapure water (PURELAB flex, ELGA LabWater, United Kingdom). Afterwards, the glass beads were further rinsed with at least 5 pore volumes of the background electrolyte solution made by adding $\mathrm{NaHCO}_{3}$ and $\mathrm{Na}_{2} \mathrm{CO}_{3}$ (Sigma-Aldrich, United States) to ultrapure water to reach a $\mathrm{pH}$ value of 9, according to the concentrations listed in Figure S1c. The experimental setup was also accurately rinsed with ultrapure water and with the considered background electrolyte solution before each experiment.

After preparing the electrolyte solutions, at least $1 \mathrm{~L}$ of such solution was poured in the glass chamber following a wet-packing procedure to minimize air entrapment ${ }^{1}$. The final size of the porous medium was $300 \mathrm{~mm} \times 180 \mathrm{~mm}$ and the water table was kept at a height of approximately $150 \mathrm{~mm}$ from the bottom of the porous medium.

Before starting the experiment, the recirculation system was activated and run for at least 30 minutes. The permanganate injection was performed using a $10 \mathrm{~mL}$ syringe filled with $8 \mathrm{~mL}$ of a $\mathrm{KMnO}_{4}$ solution $\left(3 \mathrm{mM}\right.$ ), prepared using $\mathrm{KMnO}_{4}$ powder (Sigma-Aldrich, United States) in ultrapure water. The syringe needle was modified so that the injections occurred at the same point in each experiment. pH measurements (WTW GmbH, Multi 3410, Germany) were performed at the beginning and at the end of the experiment (Table S1).

For the EK experiments, the recirculation of water to buffer electrolysis reactions at the electrodes was performed in two distinct circuits, in which the water intake from one reservoir was discharged into a $2 \mathrm{~L}$ bottle, completely filled with the same background solution of the experiment. The bottles had a sealed cap with two passing tubes, allowing to obtain equal inflow and outflow. The feed tubes, one for each bottle, were then placed in the same reservoir from which the fluid was withdrawn. The end of the tubes were placed at the bottom of the setup, in the space between the electrodes and the separators of each reservoir.

Upon application of an electric field in the EK experiments, electroosmotic flow also occurred in the glass beads porous media under the considered alkaline conditions and resulted in the displacement of water from the anode towards the cathode ${ }^{2}$. However, in the setup the development of an electroosmotic flow was quickly counterbalanced by the establishment of a hydraulic gradient in the opposite direction, due to the accumulation of water in the cathode reservoir. This resulted in a null water velocity and allowed us to investigate exclusively the effects of electromigration in the EK transport experiments. Such behavior was observed for all electrolyte concentration levels considered in this study.

The camera used to record the images during the experiments was a NIKON D300 mounting a lens AF-S NIKKOR 50mm f/1.4g, which was placed on a support $20 \mathrm{~cm}$ above the work bench and $85 \mathrm{~cm}$ from the glass chamber to align the camera sensor with respect to the center of the porous medium's section of the setup. For every experiment, the camera took pictures of the system every minute, at a resolution of $4288 \times 2848$ pixels. For every image taken the settings were: exposure time of 4 seconds, aperture of 16, ISO 200 and white balance $5000 \mathrm{~K}$.

Recorded measurements of voltage, current, $\mathrm{pH}$ and temperature for every experiment are listed in Table S1. 
a

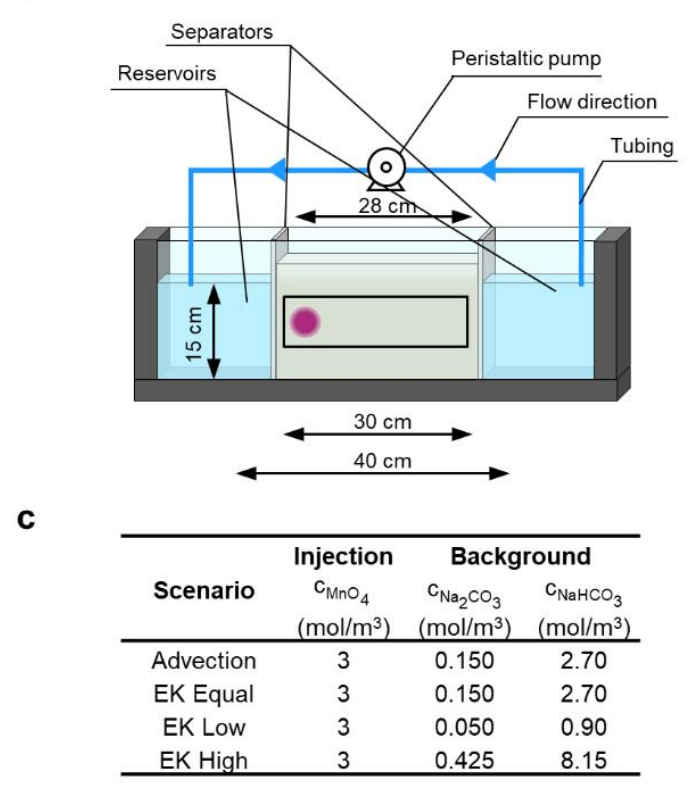

b

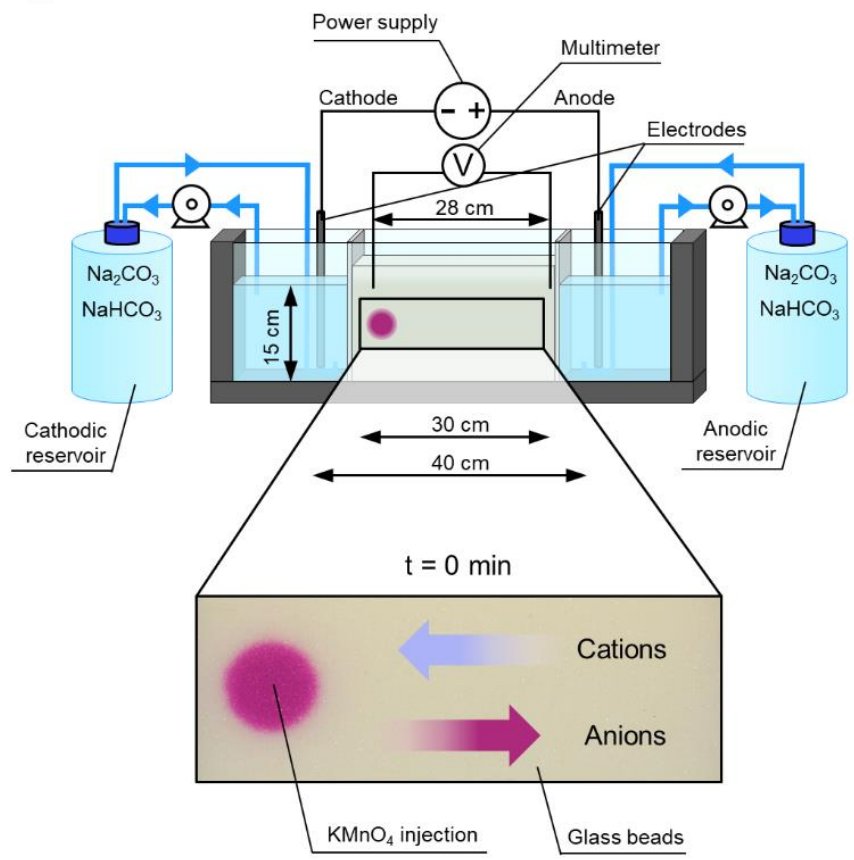

Figure S1. Illustration of experimental setup. a schematic of the setup used for advectiondispersion experiments. $\boldsymbol{b}$ setup for electrokinetic experiments. $\boldsymbol{c}$ summary of concentrations used in the different scenarios.

Table S1. Voltage, current, $p H$ and temperature in the different experiments.

\begin{tabular}{|c|c|c|c|c|c|c|}
\hline \multirow{3}{*}{ Time } & \multicolumn{3}{|c|}{ Conservative } & \multicolumn{3}{|c|}{ Reactive } \\
\hline & EK & EK & EK & EK & EK & EK \\
\hline & Equal & Low & High & Equal & Low & High \\
\hline \multicolumn{7}{|c|}{ Voltage difference between measurement electrodes (V) } \\
\hline $0 \mathrm{~h}$ & 146.0 & 148.7 & 143.8 & 146.5 & 130.0 & 143.1 \\
\hline $1 \mathrm{~h}$ & 138.4 & 131.6 & 131.4 & 139.6 & 122.9 & 132.4 \\
\hline $2 \mathrm{~h}$ & 136.3 & 127.2 & 127.5 & 136.7 & 122.3 & 127.6 \\
\hline $3 \mathrm{~h}$ & 136.2 & 123.7 & 132.8 & 135.1 & 122.1 & 131.1 \\
\hline \multicolumn{7}{|l|}{ Current (mA) } \\
\hline $0 \mathrm{~h}$ & 7.9 & 3.0 & 22.3 & 8.1 & 3.0 & 22.7 \\
\hline $1 \mathrm{~h}$ & 7.9 & 3.0 & 22.4 & 8.0 & 3.1 & 22.6 \\
\hline $2 \mathrm{~h}$ & 8.0 & 3.0 & 23.5 & 8.1 & 3.2 & 23.2 \\
\hline $3 \mathrm{~h}$ & 8.2 & 3.0 & 24.8 & 8.3 & 3.2 & 24.7 \\
\hline \multicolumn{7}{|l|}{$\mathrm{pH}$} \\
\hline $0 \mathrm{~h}$ & 9.10 & 8.88 & 9.15 & 9.02 & 8.72 & 9.17 \\
\hline $3 \mathrm{~h}$, anodic reservoir & 6.67 & 7.70 & 6.61 & 6.56 & 7.40 & 7.52 \\
\hline $3 \mathrm{~h}$, cathodic reservoir & 9.96 & 9.18 & 10.01 & 9.98 & 10.02 & 10.08 \\
\hline \multicolumn{7}{|l|}{ Temperature $\left({ }^{\circ} \mathrm{C}\right)$} \\
\hline $0 \mathrm{~h}$ & 21.3 & 21.9 & 21.7 & 21.2 & 21.6 & 21.8 \\
\hline $3 \mathrm{~h}$, anodic reservoir & 21.6 & 22.2 & 22.0 & 21.9 & 22.5 & 22.6 \\
\hline $3 \mathrm{~h}$, cathodic reservoir & 22.2 & 22.5 & 22.1 & 21.9 & 22.6 & 22.7 \\
\hline
\end{tabular}




\section{S2. Image analysis}

To relate the color intensity of permanganate in the recorded images with its concentration we performed a calibration procedure. We defined the calibration range considering the expected upper and lower values of permanganate during the experiments, ranging from 0.25 to 10.00 $\mathrm{mM}$. A schematic of the calibration process is illustrated in Figure S2a.

The calibration was conducted in the same setup used for the transport experiments. As the goal was to populate a concentration-intensity curve in the same conditions of the experiments, we prepared 13 permanganate solutions at different concentrations $(0.25,0.50,0.75,1.00,2.00$, $3.00,4.00,5.00,6.00,7.00,8.00,9.00$ and $10.00 \mathrm{mM})$. The calibration procedure was repeated for each considered concentration and consisted in the injection of $10 \mathrm{~mL}$ of $\mathrm{KMnO}_{4}$ on the right and on the center of the porous medium, forming two distinct circular plumes. In addition, a third injection of $\mathrm{KMnO}_{4}$ at reference concentration $(3 \mathrm{mM})$ was added each time on the left of the porous medium. After the three plumes were present in the domain, a picture was taken. Upon collection of all calibration images, a MATLAB script was developed to relate the color intensity with the concentrations. For every calibration image, a horizontal cross-sectional line was drawn intercepting the center of each plume (Figure S2a). Background values were also evaluated for every image. The value of each pixel was then converted from an absolute sRGB color space (0-255) to a normalized scale. Afterwards, we normalized each color intensity of the plumes on the right based on the reference intensity of the plume on the left. The relation between the injected concentrations and the normalized color intensities $\left({\overline{\langle K\rangle_{\text {norm }}}}\right)$ was then fitted with a polynomial function, $\hat{c}=f\left(\overline{\langle K}_{\text {norm }}\right)$, in which $\hat{c}$ is the estimated concentration from color intensities (Figure S2a). For every calibration image we also evaluated the standard deviation of the color intensity and we assessed the relation between such normalized intensity and its standard deviation $\sigma_{K, \text { norm }}$ for every calibration point. Subsequently, we used such function to evaluate the confidence intervals of the concentration, by considering upper and lower bounds of twice the value of the standard deviation $( \pm 2 \sigma)$. For every transport experiment we collected images every minute. The longitudinal concentration profiles were obtained averaging the color intensity values over a vertical zone of 100 pixels (green horizontal bounds in Figure S2b) and then estimating $\hat{c}$ by considering the color intensity at the considered time points normalized by the color intensity of the permanganate plume immediately after injection.

Metrics of spreading, dilution and mixing were evaluated for every image, computing $\hat{c}$ for every pixel and subsequently evaluating Eq. 9-14. 
a
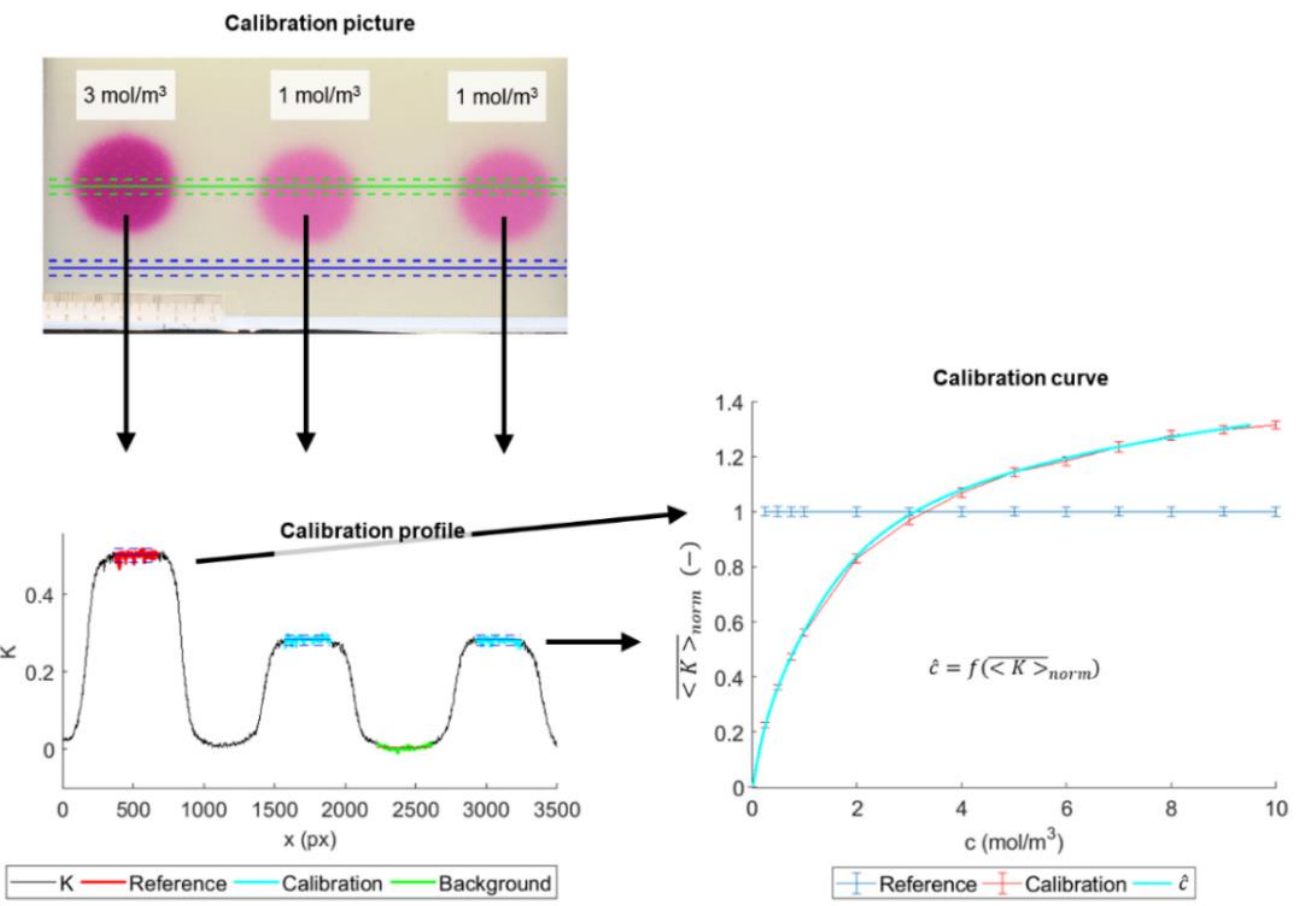

b

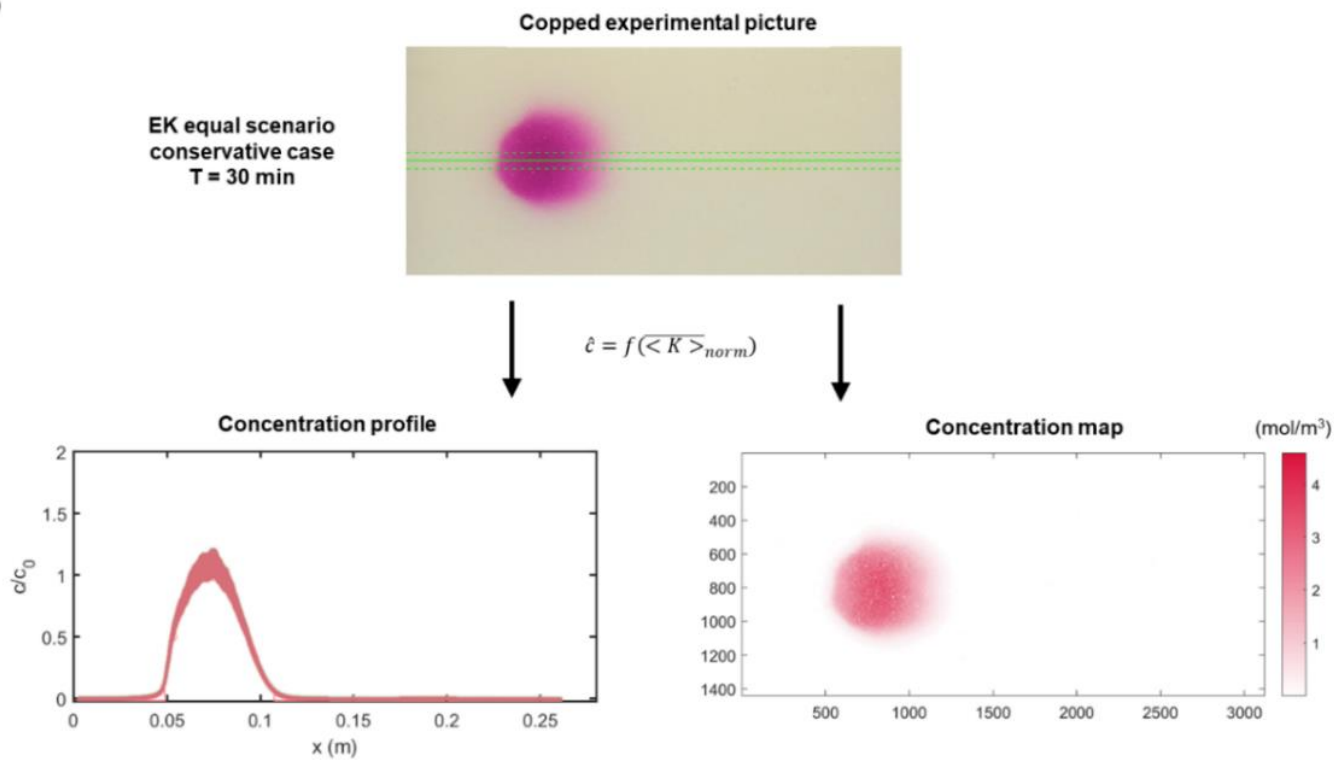

Figure S2. a Illustration of the steps for the calibration procedure. The calibration picture presents a reference injection of permanganate of $3 \mathrm{~mol} / \mathrm{m}^{3}$ on the left and two calibration spots of $1 \mathrm{~mol} / \mathrm{m}^{3}$ on the center and right of the domain. Calibration profiles are evaluated for all investigated permanganate solutions and results are used for the calibration and standard deviation curves. $\boldsymbol{b}$ Illustration of the image analysis procedure for the scenario "EK equal", considering the picture taken at $t=30$ minutes. 


\section{S3. Governing equations of charged species transport}

The transport of charged species in porous media can be described by the following expression of the Nernst-Planck equation:

$$
\boldsymbol{J}_{i}^{T o t}=\underbrace{-n D_{i} \nabla c_{i}}_{\boldsymbol{J}_{i}^{\text {Dif }}} \underbrace{-n D_{i} \frac{Z_{i} F}{R T} c_{i} \nabla \Phi}_{\boldsymbol{J}_{i}^{\text {Mig }}} \underbrace{+n \boldsymbol{u} c_{i}}_{\boldsymbol{J}_{i}^{\text {A } d v}}
$$

where $n$ is the accessible porosity, $D_{i}$ is the pore diffusion coefficient $\left(D_{i}=D_{i}^{a q} \tau\right.$ with $D_{i}^{a q}$ being the aqueous molecular diffusion coefficient of the dissolved species $i$ and $\tau$ the tortuosity), $\nabla c_{i}$ is the concentration gradient, $z_{i}$ the charge of the species $i, F$ the Faraday constant, $R$ the gas constant, $T$ the temperature, $c_{i}$ the molar concentration, $\nabla \Phi$ the electric potential gradient and $\boldsymbol{u}$ is the seepage velocity.

In case of advective-dispersive transport, the hydrodynamic dispersion coefficients are described with the following parameterizations for the longitudinal and transverse direction, respectively ${ }^{3,4}$ :

$$
\begin{gathered}
D_{i}^{L}=D_{i}+0.5 u d \\
D_{i}^{T}=D_{i}+D_{i}^{a q}\left(\frac{P e_{i}^{2}}{P e_{i}+2+4 \delta^{2}}\right)^{\beta}
\end{gathered}
$$

where $d$ is the average grain size diameter of the porous medium, $P e_{i}=u d / D_{i}^{a q}$ is the grain Péclet number and $u$ is the seepage velocity along the main flow direction. $\delta$ indicates the ratio between the length of a pore channel and its hydraulic radius, and $\beta$ is an empirical exponent accounting for the effect of incomplete mixing in the pore channels. The values of these parameters, $\delta=5.37$ and $\beta=0.5$, have been determined in previous studies ${ }^{5,6}$.

The material balance for a species $i$ yields the governing multicomponent ionic transport equation:

$$
\frac{\partial\left(n c_{i}\right)}{\partial t}+\nabla \cdot \boldsymbol{J}_{i}^{T o t}=r_{i}
$$

in which $r_{i}$ is the source/sink term.

The relation between charge density of the solution $\left(\rho_{e}\right)$ and electric field is described by the Poisson's equation ${ }^{7}$ :

$$
\nabla^{2} \Phi=-\frac{F}{\varepsilon} \sum_{i=1}^{N} z_{i} c_{i}=-\frac{\rho_{e}}{\varepsilon}
$$

where $\varepsilon$ is the dielectric constant of the porous medium and $N$ is the number of charged species in solution.

In electrolyte systems, the current can be assumed to be carried by ions in solutions ${ }^{7}$, therefore, it is possible to express the current density $I$ as the total flux of the ions in the system:

$$
\boldsymbol{I}=F \sum_{i=1}^{N} z_{i} \boldsymbol{J}_{i}^{T o t}=-F \sum_{i=1}^{N} z_{i} n D_{i} \nabla c_{i}-\left(F^{2} \sum_{i=1}^{N} z_{i}^{2} \frac{n D_{i}}{R T} c_{i}\right) \nabla \Phi+F n \boldsymbol{v}_{\boldsymbol{e}} \sum_{i=1}^{N} z_{i} c_{i}
$$


The continuity condition in terms of charge can be expressed as:

$$
F \sum_{i=1}^{N} z_{i} \frac{\partial\left(n c_{i}\right)}{\partial t}+\nabla \cdot\left(F \sum_{i=1}^{N} z_{i} \boldsymbol{J}_{i}^{T o t}\right)=F \sum_{i=1}^{N} z_{i} r_{i}
$$

The set of Eq. 1 and 4-7 constitutes the Nernst-Planck-Poisson equations (NPPE) and describes the transport of charged species considering charge interactions. The reaction term $r_{i}$ for permanganate degradation is described as a complete bimolecular reaction:

$$
r_{\mathrm{MnO}_{4}^{-}}=k_{r, \mathrm{MnO}_{4}^{-}} C_{\mathrm{MnO}_{4}^{-}} c_{\mathrm{C}_{6} \mathrm{H}_{12} \mathrm{O}_{6}}
$$

in which $k_{r, M n O}-$ is the kinetic rate constant (Table S2).

\section{S4. Metrics of spreading, dilution and mixing}

The distribution of solute concentration can be characterized using spatial moments. The zeroth moment indicates the total mass of solute $i$ in a 2-D system with total area $\Omega$ :

$$
M_{0, i}(t)=\int_{\Omega} c_{i}(\boldsymbol{x}, t) d \Omega
$$

The first-order moment in the $m^{\text {th }}$ direction divided by the total mass yields the location of the plume centroid in a given $m$ direction:

$$
\bar{x}_{i, m}(t)=\frac{1}{M_{0, i}(t)} \int_{\Omega} c_{i}(\boldsymbol{x}, t) x_{m} d \Omega
$$

The second central moment, represents the mean square displacement from the plume centroid in directions $m$ and $n$ :

$$
M_{i, 2, m n}=\int_{\Omega} c_{i}(\boldsymbol{x}, t)\left(x_{m}-\bar{x}_{i, m}(t)\right)\left(x_{n}-\bar{x}_{i, n}(t)\right) d \Omega
$$

Measurements of spreading and dispersion of the plume can be evaluated considering the temporal evolution of $M_{i, 2, m n} / M_{0, i}{ }^{8}$.

The dilution index is a metric that quantifies dilution as the act of distributing the mass of a solute over a larger volume (area in 2-D) and is a measure of the effective volume (area in 2D) of a dissolved plume. It is defined as the exponent of the Shannon entropy ${ }^{9}$ :

$$
E(t)=\exp \left(-\int_{\Omega} p_{i}(\boldsymbol{x}, t) \ln \left(p_{i}(\boldsymbol{x}, t)\right) d \Omega\right)
$$

in which $p_{i}(\boldsymbol{x}, t)$ is the concentration probability distribution:

$$
p_{i}(\boldsymbol{x}, t)=\frac{c_{i}(\boldsymbol{x}, t)}{\int_{\Omega} c_{i}(\boldsymbol{x}, t) d \Omega}
$$

As a measure of reactive mixing we calculate the mixing area $A_{\text {mix }}$, which considers the area of the domain in which the reaction rate exceeds a threshold:

$$
A_{\text {mix }}=\int_{\Omega} H\left(r_{\mathrm{MnO}_{4}}(\boldsymbol{x}, t)-r_{\text {lim }}\right) d \Omega
$$

where $H$ is the Heaviside step function and $r_{l i m}$ is the limiting reaction rate threshold, set in this study to $r_{\text {lim }}=2 \times 10^{-5} \mathrm{~mol} / \mathrm{m}^{3} / \mathrm{s}$. 
The remaining mass of permanganate was evaluated for the reactive transport cases to quantify the efficiency of the mixing-controlled degradation in the different scenarios and was calculated with Eq. 9.

\section{S5. Numerical Modeling}

The model domain for all scenarios was a rectangular area with size $280 \mathrm{~mm} \times 150 \mathrm{~mm}$, discretized with 13400 triangular elements (Figure S3). The model simulated the saturated porous medium and was defined for a length of $280 \mathrm{~mm}$, spanning the distance between the measurement electrodes, where the electric potential was recorded.

The initial injection zone of the tracer was modeled considering a circle of radius $21.5 \mathrm{~mm}$. Dirichlet boundary conditions were set for the concentration at the sides of the domain, with concentrations equal to the background for each scenario. The electric potential at the boundaries was set to the measured values during the experiments (Table S1). Electrolysis reactions were not included in the model due to the large reservoir volumes employed to buffer such reactions and to the use of a carbonate buffer solution as electrolyte, which minimized the impact of $\mathrm{pH}$ changes in the domain.

The only fitting parameter for the simulation of the EK experiments was the tortuosity, which was found to vary in a range 0.50-0.60. Values of the other input model parameters are listed in Table S2.

Aqueous diffusion coefficients were evaluated at a temperature of $22{ }^{\circ} \mathrm{C}$ (Table S3) and initial concentration values for the aqueous species included in the model have been obtained after speciation reaction of $\mathrm{NaHCO}_{3}$ and $\mathrm{Na}_{2} \mathrm{CO}_{3}$ (Table S4).

Surface integration calculations for the quantification of spreading, dilution and mixing were evaluated considering permanganate concentrations larger than $0.04 \mathrm{~mol} / \mathrm{m}^{3}$, which is the threshold used in the image analysis procedure.

The simulated plumes of $\mathrm{MnO}_{4}^{-}$in the domain for the different experimental scenarios at different times are illustrated in Figure S4, whereas Figure S5 and Figure S6 present information regarding the order of magnitude and spatial distribution of advective, diffusive/dispersive and electromigration fluxes.

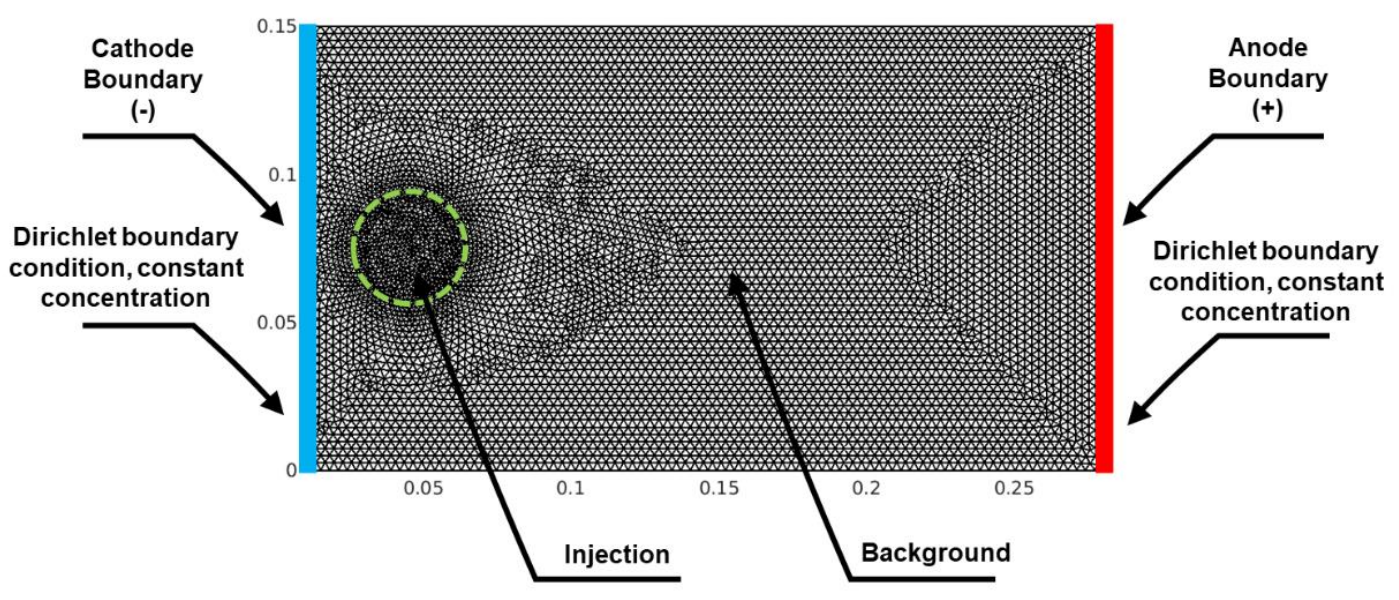

Figure S3. Mesh used in the model simulations (13400 triangular elements) with indication of domains and boundary conditions. 
Table S2. Input model parameters.

\begin{tabular}{|c|c|c|c|c|c|c|c|c|c|}
\hline & & \multicolumn{4}{|c|}{ Conservative } & \multicolumn{4}{|c|}{ Reactive } \\
\hline & \multirow[b]{2}{*}{ Units } & \multicolumn{2}{|r|}{ EK } & \multirow{2}{*}{$\begin{array}{c}\text { EK } \\
\text { Low }\end{array}$} & \multirow{2}{*}{$\begin{array}{c}\text { EK } \\
\text { High }\end{array}$} & \multicolumn{3}{|c|}{ EK } & \multirow{2}{*}{$\begin{array}{c}\text { EK } \\
\text { High }\end{array}$} \\
\hline & & $\mathrm{AD}$ & Equal & & & $\mathrm{AD}$ & Equal & EK Low & \\
\hline Porosity & - & 0.40 & 0.40 & 0.40 & 0.40 & 0.40 & 0.40 & 0.40 & 0.40 \\
\hline Tortuosity & - & 0.61 & 0.61 & 0.51 & 0.56 & 0.61 & 0.61 & 0.51 & 0.56 \\
\hline \multicolumn{10}{|c|}{ Electric potential at the anode } \\
\hline $0 \mathrm{~h}$ & $\mathrm{~V}$ & - & 146 & 148.7 & 143.8 & - & 146.5 & 130 & 143.1 \\
\hline $1 \mathrm{~h}$ & V & - & 138.4 & 131.6 & 131.4 & - & 139.6 & 122.9 & 132.4 \\
\hline $2 \mathrm{~h}$ & V & - & 136.3 & 127.2 & 127.5 & - & 136.7 & 122.3 & 127.6 \\
\hline $3 \mathrm{~h}$ & $\mathrm{~V}$ & - & 136.2 & 123.7 & 132.8 & - & 135.1 & 122.1 & 131.1 \\
\hline $\begin{array}{l}\text { Seepage } \\
\text { velocity }\end{array}$ & $\mathrm{cm} / \mathrm{h}$ & 6.5 & 0 & 0 & 0 & 6.5 & 0 & 0 & 0 \\
\hline $\begin{array}{l}\text { Kinetic rate } \\
\text { constant } \\
\left(k_{r, M n O_{4}^{-}}\right)\end{array}$ & $\mathrm{m}^{3} / \mathrm{s} / \mathrm{mol}$ & 0 & 0 & 0 & 0 & $8 \times 10^{-6}$ & $8 \times 10^{-6}$ & $8 \times 10^{-6}$ & $8 \times 10^{-6}$ \\
\hline
\end{tabular}

Table S3. Aqueous diffusion coefficients of the species used in the model at $22{ }^{\circ} \mathrm{C}$.

\begin{tabular}{ll}
\hline $\begin{array}{l}\text { Diffusion coefficients }(22 \\
\left.{ }^{\circ} \mathrm{C}\right)\end{array}$ & $\left(\mathrm{m}^{2} / \mathrm{s}\right)$ \\
\hline $\mathrm{H}^{+}$ & $8.59 \times 10^{-9}$ \\
$\mathrm{OH}^{-}$ & $4.86 \times 10^{-9}$ \\
$\mathrm{Na}^{+}$ & $1.23 \times 10^{-9}$ \\
$\mathrm{CO}_{3}{ }^{2-}$ & $8.82 \times 10^{-10}$ \\
$\mathrm{HCO}_{3}{ }^{-}$ & $1.09 \times 10^{-9}$ \\
$\mathrm{MnO}_{4}^{-}$ & $1.50 \times 10^{-9}$ \\
$\mathrm{NaCO}_{3}{ }^{-}$ & $1.11 \times 10^{-9}$ \\
$\mathrm{NaHCO}_{3}$ & $6.21 \times 10^{-10}$ \\
$\mathrm{~K}^{+}$ & $1.81 \times 10^{-9}$ \\
$\mathrm{Glucose}$ & $6.19 \times 10^{-10}$ \\
\hline
\end{tabular}

Table S4. Initial values of the background solution used in the different scenarios.

\begin{tabular}{lcccc}
\hline & Units & $\begin{array}{c}\text { Advection- } \\
\text { dispersion and } \\
\text { EK Equal }\end{array}$ & EK Low & EK High \\
\hline Temperature & ${ }^{\circ} \mathrm{C}$ & 22 & 22 & 22 \\
$\mathrm{pH}$ & - & 9.01 & 9.00 & 8.96 \\
$\mathrm{Alkalinity}$ & $\mathrm{eq} / \mathrm{L}$ & $3.01 \times 10^{-3}$ & $1.00 \times 10^{-3}$ & $9.09 \times 10^{-3}$ \\
$\mathrm{OH}^{-}$ & $\mathrm{mol} / \mathrm{m}^{3}$ & $8.86 \times 10^{-6}$ & $8.44 \times 10^{-6}$ & $8.14 \times 10^{-6}$ \\
$\mathrm{H}^{+}$ & $\mathrm{mol} / \mathrm{m}^{3}$ & $1.02 \times 10^{-9}$ & $1.02 \times 10^{-9}$ & $1.19 \times 10^{-9}$ \\
$\mathrm{HCO}_{3}{ }^{-}$ & $\mathrm{mol} / \mathrm{m}^{3}$ & $2.69 \times 10^{-3}$ & $9.04 \times 10^{-4}$ & $8.09 \times 10^{-3}$ \\
$\mathrm{CO}_{3}{ }^{2-}$ & $m o l / \mathrm{m}^{3}$ & $1.47 \times 10^{-4}$ & $4.49 \times 10^{-5}$ & $4.36 \times 10^{-4}$ \\
$\mathrm{NaCO}_{3}{ }^{-}$ & $\mathrm{mol} / \mathrm{m}^{3}$ & $5.57 \times 10^{-6}$ & $6.24 \times 10^{-7}$ & $4.27 \times 10^{-5}$ \\
$\mathrm{NaHCO}_{3}$ & $\mathrm{~mol} / \mathrm{m}^{3}$ & $4.10 \times 10^{-6}$ & $4.83 \times 10^{-7}$ & $3.42 \times 10^{-5}$ \\
$\mathrm{Na}^{+}$ & $\mathrm{mol} / \mathrm{m}^{3}$ & $3.00 \times 10^{-3}$ & $1.00 \times 10^{-3}$ & $9.01 \times 10^{-3}$ \\
\hline
\end{tabular}



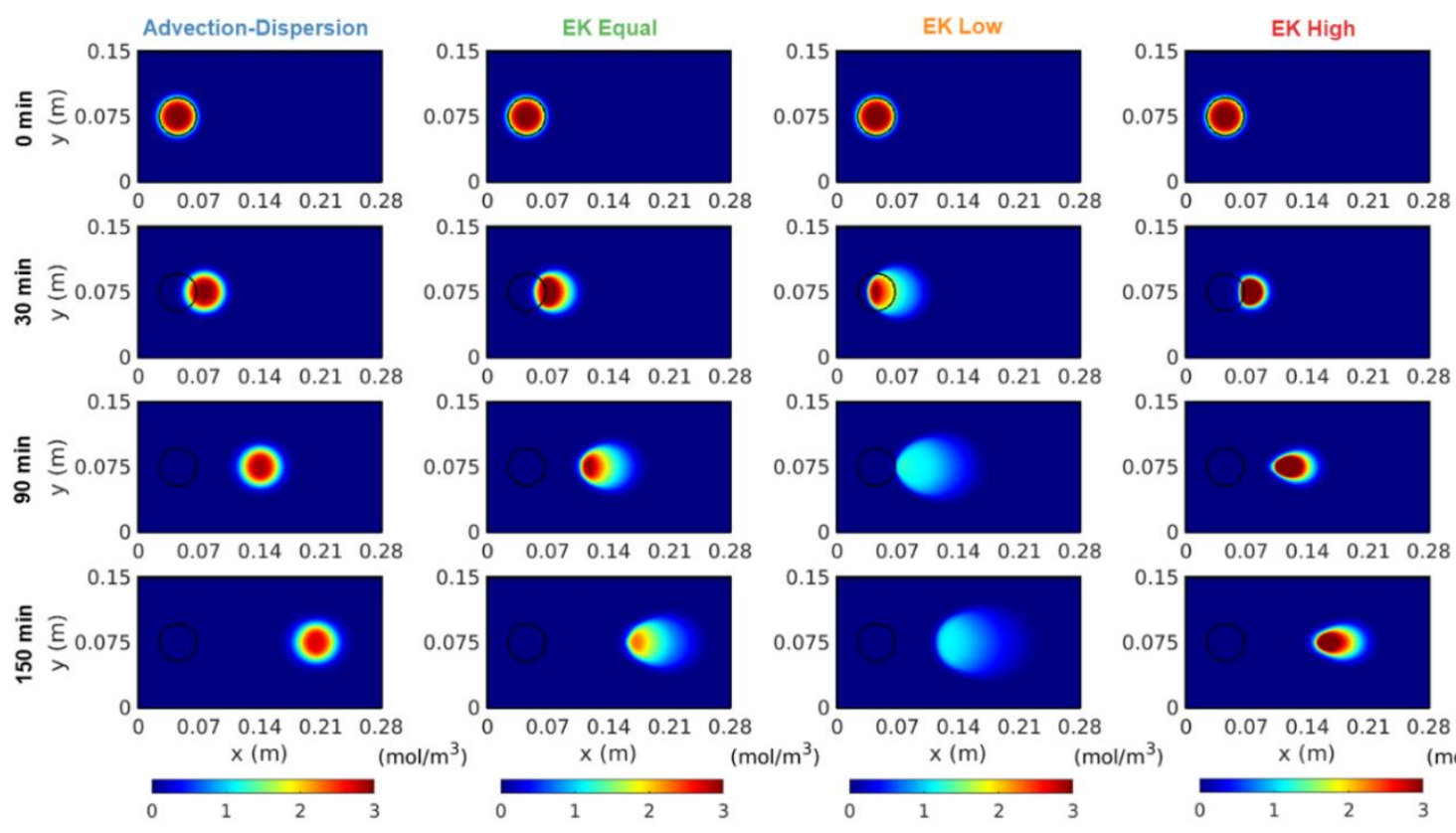

Figure S4. Simulated concentration of $\mathrm{MnO}_{4}^{-}$at different times for the four conservative scenarios.
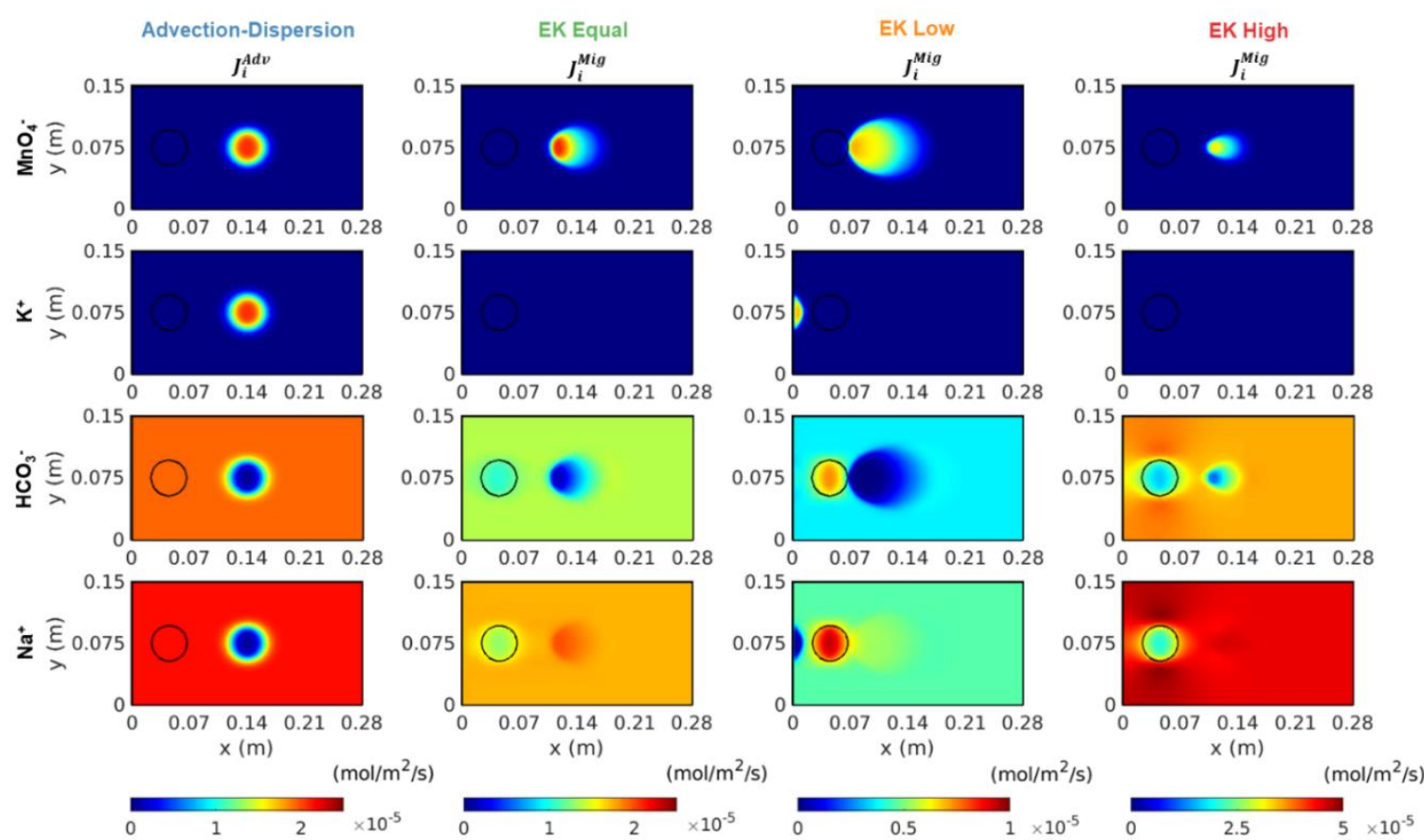

Figure S5. Simulated advective and electromigration fluxes for the four scenarios at 90 minutes. 

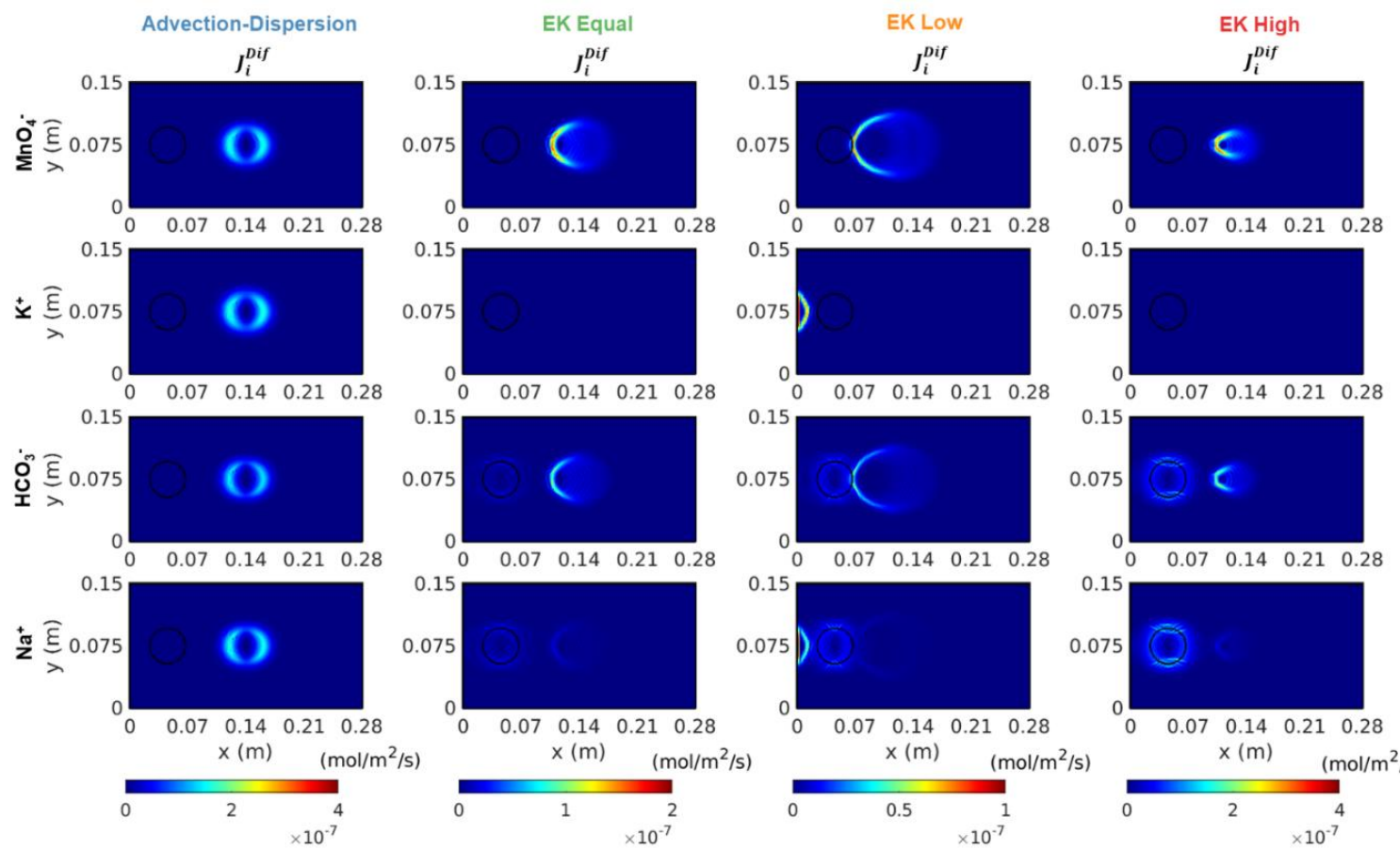

Figure S6. Simulated diffusive/dispersive fluxes for the four scenarios at 90 minutes. 


\section{SI References}

(1) Haberer, C. M.; Rolle, M.; Cirpka, O. A.; Grathwohl, P. Oxygen Transfer in a Fluctuating Capillary Fringe. Vadose Zo. J. 2012, 11 (3).

https://doi.org/10.2136/vzj2011.0056.

(2) Qin, J.; Sun, X.; Liu, Y.; Berthold, T.; Harms, H.; Wick, L. Y. Electrokinetic Control of Bacterial Deposition and Transport. Environ. Sci. Technol. 2015, 49 (9), 56635671. https://doi.org/10.1021/es506245y.

(3) Guedes De Carvalho, J. R. F.; Delgado, J. M. P. Q. Overall Map and Correlation of Dispersion Data for Flow through Granular Packed Beds. Chem. Eng. Sci. 2005, 60 (2), 365-375. https://doi.org/10.1016/j.ces.2004.07.121.

(4) Rolle, M.; Hochstetler, D.; Chiogna, G.; Kitanidis, P. K.; Grathwohl, P. Experimental Investigation and Pore-Scale Modeling Interpretation of Compound-Specific Transverse Dispersion in Porous Media. Transp. Porous Media 2012, 93 (3), 347-362. https://doi.org/10.1007/s11242-012-9953-8.

(5) Hochstetler, D. L.; Rolle, M.; Chiogna, G.; Haberer, C. M.; Grathwohl, P.; Kitanidis, P. K. Effects of Compound-Specific Transverse Mixing on Steady-State Reactive Plumes: Insights from Pore-Scale Simulations and Darcy-Scale Experiments. Adv. Water Resour. 2013, 54, 1-10. https://doi.org/10.1016/j.advwatres.2012.12.007.

(6) Ye, Y.; Chiogna, G.; Cirpka, O.; Grathwohl, P.; Rolle, M. Experimental Investigation of Compound-Specific Dilution of Solute Plumes in Saturated Porous Media: 2-D vs. 3-D Flow-through Systems. J. Contam. Hydrol. 2015, 172, 33-47. https://doi.org/10.1016/j.jconhyd.2014.11.002.

(7) Newman, J.; Thomas-Alyea, K. E. Electrochemical Systems; John Wiley \& Sons, 2004.

(8) Kitanidis, P. K. Analysis of Macrodispersion through Volume-Averaging: Moment Equations. Stoch. Hydrol. Hydraul. 1992, 6 (1), 5-25.

https://doi.org/10.1007/BF01581672.

(9) Kitanidis, P. K. The Concept of the Dilution Index. Water Resour. Res. 1994, 30 (7), 2011-2026. https://doi.org/10.1029/94WR00762.

(10) Rolle, M.; Sprocati, R.; Masi, M.; Jin, B.; Muniruzzaman, M. Nernst-Planck-Based Description of Transport, Coulombic Interactions, and Geochemical Reactions in Porous Media: Modeling Approach and Benchmark Experiments. Water Resour. Res. 2018, 54 (4), 3176--3195. https://doi.org/10.1002/2017WR022344.

(11) Sprocati, R.; Masi, M.; Muniruzzaman, M.; Rolle, M. Modeling Electrokinetic Transport and Biogeochemical Reactions in Porous Media: A Multidimensional Nernst-Planck-Poisson Approach with PHREEQC Coupling. Adv. Water Resour. 2019, 127, 134-147. https://doi.org/10.1016/j.advwatres.2019.03.011.

(12) Parkhurst, D. L.; Wissmeier, L. PhreeqcRM: A Reaction Module for Transport Simulators Based on the Geochemical Model PHREEQC. Adv. Water Resour. 2015, 83, 176-189. https://doi.org/10.1016/j.advwatres.2015.06.001. 